\title{
Effects of strain on orbital ordering and magnetism at perovskite oxide interfaces: $\mathrm{LaMnO}_{3} / \mathrm{SrMnO}_{3}$
}

\author{
B. R. K. Nanda and Sashi Satpathy \\ Department of Physics and Astronomy, University of Missouri, Columbia, Missouri 65211, USA \\ (Received 31 March 2008; revised manuscript received 8 July 2008; published 19 August 2008)
}

\begin{abstract}
We study how strain affects orbital ordering and magnetism at the interface between $\mathrm{SrMnO}_{3}$ and $\mathrm{LaMnO}_{3}$ from density-functional calculations and interpret the basic results in terms of a three-site Mn-O-Mn model. Magnetic interaction between the $\mathrm{Mn}$ atoms is governed by a competition between the antiferromagnetic superexchange of the $\mathrm{Mn} t_{2 g}$ core spins and the ferromagnetic double exchange of the itinerant $e_{g}$ electrons. While the core electrons are relatively unaffected by the strain, the orbital character of the itinerant electron is strongly affected, which in turn causes a large change in the strength of the ferromagnetic double exchange. The epitaxial strain produces the tetragonal distortion of the $\mathrm{MnO}_{6}$ octahedron, splitting the $\mathrm{Mn} e_{g}$ states into $x^{2}-y^{2}$ and $3 z^{2}-1$ states, with the former being lower in energy, if the strain is tensile in the plane and opposite if the strain is compressive. For the case of the tensile strain, the resulting higher occupancy of the $x^{2}-y^{2}$ orbital enhances the in-plane ferromagnetic double exchange owing to the larger electron hopping in the plane, causing at the same time a reduction in the out-of-plane double exchange. This reduction is large enough to be overcome by antiferromagnetic superexchange, which wins to produce a net antiferromagnetic interaction between the out-of-plane Mn atoms. For the case of the in-plane compressive strain, the reverse happens, viz., that the higher occupancy of the $3 z^{2}-1$ orbital results in the out-of-plane ferromagnetic interaction, while the in-plane magnetic interaction remains antiferromagnetic. Concrete density-functional results are presented for the $\left(\mathrm{LaMnO}_{3}\right)_{1} /\left(\mathrm{SrMnO}_{3}\right)_{1}$ and $\left(\mathrm{LaMnO}_{3}\right)_{1} /\left(\mathrm{SrMnO}_{3}\right)_{3}$ superlattices for various strain conditions.
\end{abstract}

DOI: 10.1103/PhysRevB.78.054427

PACS number(s): 75.70.Cn, 71.20.-b, 73.20.-r, 71.70.-d

\section{INTRODUCTION}

Recent advances in successfully designing atomically sharp interfaces between dissimilar transition-metal oxides have revealed the formation of new electronic and magnetic phases at the vicinity of the interface, which are qualitatively different from the parent compounds. The interfacial phases show diverse magnetic properties due to the coupling between charge, orbital, and spin degrees of freedom. For example, the magnetic ordering at the interface between the two antiferromagnetic (AFM) insulators $\mathrm{SrMnO}_{3}(\mathrm{SMO})(\mathrm{G}$ type) and $\mathrm{LaMnO}_{3}$ (LMO) (A type), schematically shown in Fig. 1, could be ferromagnetic (FM) along all directions, ferromagnetic in the $x y$ plane and antiferromagnetic normal to the plane or antiferromagnetic in the plane and ferromagnetic normal to the plane depending on the composition of the parent compounds and epitaxial strain on the interface. ${ }^{1-6}$

The epitaxial strain, arising due to lattice mismatch between the constituent compounds of the superlattice and the substrate, induces anisotropic hopping between orbitals to cause orbital ordering at the interface. By varying the strain condition the orbital ordering changes which in turn changes the magnetic ordering at the interface. In this paper we examine the magnetic properties at the interface of $\mathrm{SrMnO}_{3}$ and $\mathrm{LaMnO}_{3}$ for different epitaxial strain conditions through first-principles electronic structure calculations.

Experimental studies show that if the substrate induces tensile strain at the interface of the LMO/SMO superlattice, where the in-plane lattice parameter $a$ is greater than the out-of-plane lattice parameter $c$, as in the case of $(\mathrm{LMO})_{3} /(\mathrm{SMO})_{2}$ superlattice grown on $\mathrm{SrTiO}_{3}(\mathrm{STO})$ substrate, the magnetic ordering of the interfacial $\mathrm{Mn}$ atoms is
A type with in-plane $\left(\mathrm{MnO}_{2}\right.$ plane $) \mathrm{FM}$ ordering and out-ofplane (between $\mathrm{MnO}_{2}$ planes) AFM ordering. ${ }^{1}$

Quite interestingly, when the $(\mathrm{LMO})_{3} /(\mathrm{SMO})_{2}$ superlattice is grown on $\mathrm{La}_{0.3} \mathrm{Sr}_{0.7} \mathrm{Al}_{0.65} \mathrm{Ta}_{0.35} \mathrm{O}_{3}$ (LSAT) substrate, which induces no strain $(a \sim c)$, the interface shows a three-

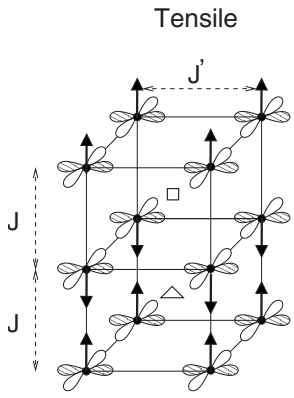

A

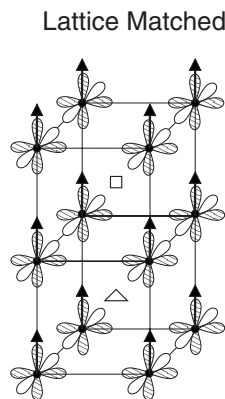

$\mathrm{F}$ $d_{x^{2}-y^{2}}$

$$
d^{2}-y^{2}+d_{3 z^{2}-1}
$$

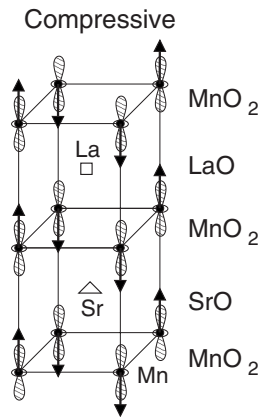

C

$\mathrm{d}_{3 z^{2}-1}$
FIG. 1. Different magnetic configurations considered in this paper for the $(\mathrm{LMO})_{1} /(\mathrm{SMO})_{1}$ superlattice. A stands for the structure with ferromagnetic ordering in the $\mathrm{MnO}_{2}$ plane and antiferromagnetic ordering between the planes, while " $F$ " stands for ferromagnetic ordering in all directions and " $\mathrm{C}$ " stands for antiferromagnetic ordering in the $\mathrm{MnO}_{2}$ plane and ferromagnetic ordering between the planes. The schematic orbital ordering shown in the figure was found from our density-functional results presented below and was also inferred from the experiments (Ref. 1). The symbols $J$ and $J^{\prime}$ denote, respectively, the out-of-plane and in-plane exchange interactions between the Mn atoms. The strain condition under which each structure is stabilized has been indicated in the figure. The oxygen atoms which occur at the midpoint between two neighboring $\mathrm{Mn}$ atoms have not been shown. 


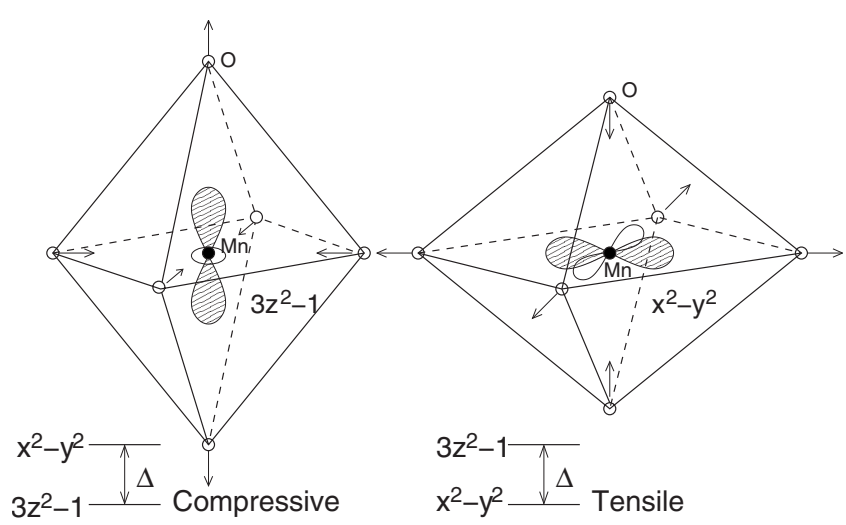

FIG. 2. Energy splitting of the $\operatorname{Mn}\left(e_{g}\right)$ orbitals at the LMO/SMO interface for compressive and tensile strain conditions. The parameter $\Delta$ is the difference between the energies of the $\mathrm{Mn} d_{x^{2}-y^{2}}$ and the $\mathrm{Mn} d_{3 z^{2}-1}$ orbitals. Compressive strain makes the $3 z^{2}-1$ orbital lower in energy, while tensile strain makes it higher.

dimensional FM ordering (F type). ${ }^{1}$ If the interface experiences a compressive strain $(a<c)$, as in the case of LMO/ SMO superlattice grown on $\mathrm{LaAlO}_{3}$ (LAO) substrate, the magnetic ordering is $\mathrm{C}$ type with in-plane AFM ordering and out-of-plane FM ordering. ${ }^{1}$

Substrates are instrumental in inducing epitaxial strain and thereby enforce tetragonal distortion to the superlattice. As a consequence, in case of LMO/SMO superlattice, the substrate distorts the $\mathrm{MnO}_{6}$ octahedron and splits the degenerate $\mathrm{Mn} e_{g}$ states into $x^{2}-y^{2}$ and $3 z^{2}-1$ states. Varied tetragonal distortion changes the on-site energy and hence the occupancy of these two nondegenerate $e_{g}$ states (Fig. 2). Since the electronic configuration of $\mathrm{Mn}$ atoms away from the interface is the same as in the bulk compounds, $\left(\mathrm{Mn}^{4+}, t_{2 g}^{3} e_{g}^{0}\right)$ for SMO and $\left(\mathrm{Mn}^{3+}, t_{2 g}^{3} e_{g}^{1}\right)$ for LMO, strain is not expected to affect the magnetic configuration of the inner $\mathrm{MnO}_{2}$ layers to a large extent. However, at the interface, where we see the valence state of the $\mathrm{Mn}$ atoms lies between $3+$ and $4+$ because of charge reconstruction, ${ }^{2,7}$ the varied occupancy of the nondegenerate $e_{g}$ orbitals imposes different orbital orderings for different strain conditions (Fig. 2) and influences the interface magnetism considerably.

In this paper, we have studied in detail the interfacial magnetic properties of LMO/SMO superlattices for different strain conditions by performing electronic structure calculations based on the density-functional theory (DFT). To illustrate the strain effect on magnetism, we have proposed a simple three-site model to calculate the interfacial Mn-O-Mn magnetic exchange both in the $\mathrm{MnO}_{2}$ plane and between the planes for different strain conditions. From the model we see that the on-site energy difference between $x^{2}-y^{2}$ and $3 z^{2}-1$ orbitals (Fig. 2) is instrumental in switching the ferromagnetic and antiferromagnetic interactions. When the $3 z^{2}-1$ orbital is sufficiently lower in energy than the $x^{2}-y^{2}$ orbital (compressive strain), the Mn-O-Mn exchange is antiferromagnetic in the plane and ferromagnetic between the planes and opposite when $x^{2}-y^{2}$ orbital is sufficiently lower in energy (tensile strain). If the energy levels of both the $e_{g}$ orbitals are close enough (lattice-matched interface), then the $\mathrm{Mn}-\mathrm{O}-\mathrm{Mn}$ exchange is ferromagnetic in all directions.
The rest of the paper is organized as follows. In Sec. II we describe the structural and computational details. A detailed analysis of the electronic structure of the $(\mathrm{LMO})_{1} /(\mathrm{SMO})_{1}$ superlattice at different strain conditions, obtained from the density-functional calculations, is carried out in Sec. III. In Sec. IV, we illustrate the effect of epitaxial strain on the magnetic ordering, with the aid of a proposed three-site $(\mathrm{Mn}$ $\mathrm{O}-\mathrm{Mn})$ model. Electronic and magnetic properties of the $(\mathrm{LMO})_{1} /(\mathrm{SMO})_{3}$ superlattice at different strain conditions are discussed in Sec. V. Finally in Sec. VI we present the summary.

\section{STRUCTURAL AND COMPUTATIONAL DETAILS}

We have taken the equivalent cubic perovskite structure of LMO and SMO in order to study the electronic and magnetic properties at the interface of these two compounds with the aid of first-principles electronic structure calculations. The effect of epitaxial strain, which arises due to lattice mismatch between the substrate and the LMO/SMO superlattice, is taken into account by applying tetragonal distortion to the superlattice.

The tetragonal distortion is quantified by the $c / a$ ratio which differs from one. Here $a$ is the in-plane ( $x y$-plane) lattice parameter which coincides with the lattice parameter of the substrate and $c$ is the average out-of-plane lattice parameter (along $z$ axis). The $c / a$ ratio is determined from the linear relation: $c-a_{0}=-4 \nu\left(a-a_{0}\right)$, where $a_{0}$ is the in-plane lattice parameter of the superlattice when there is no strain $(c / a=1)$ and coefficient $\nu$ is the Poisson ratio which is approximately 0.3 for perovskite manganites. ${ }^{1,8}$ Experimentally it is found that for LMO/SMO superlattices, $a_{0}$ matches with the weighed average of the lattice constants of bulk LMO (3.936 $\AA$ ) and bulk SMO (3.806 ̊). ${ }^{1}$ For example, for $(\mathrm{LMO})_{1} /(\mathrm{SMO})_{1}$ superlattice, the value of $a_{0}$ is $\frac{1}{2}(3.936+3.806) \AA$.

In this paper, we have considered two superlattices, viz., $(\mathrm{LMO})_{1} /(\mathrm{SMO})_{1}$ and $(\mathrm{LMO})_{1} /(\mathrm{SMO})_{3}$, to study the electronic and magnetic properties at different strain conditions. As is well known, the strength of the Jahn-Teller (JT) distortion is less in the mixed compounds ( $\mathrm{La}, \mathrm{Sr}) \mathrm{MnO}_{3}$ as compared to that of $\mathrm{LaMnO}_{3}$, we have considered a small JahnTeller distortion $\left(Q_{2} \approx 0.05 \AA\right)$ in the basal plane for the interfacial $\mathrm{MnO}_{2}$ layers. However, test calculations showed that a small variation of $Q_{2}$ does not change the electronic and magnetic properties of the superlattice qualitatively.

All electronic structure calculations reported in this work have been performed using the self-consistent tight-binding linearized muffin-tin orbital (TB-LMTO) method with the atomic sphere approximation (ASA). ${ }^{9}$ Self-consistent calculations are done within the framework of generalized gradient approximation including Coulomb correction $(\mathrm{GGA}+U)$. All results are obtained with $U=5 \mathrm{eV}$ and $J=1 \mathrm{eV}$ unless otherwise stated.

\section{ELECTRONIC STRUCTURE OF THE $\left(\mathrm{LaMnO}_{3}\right)_{1} /\left(\mathrm{SrMnO}_{3}\right)_{1}$ SUPERLATTICE}

In this section, we describe the effect of strain on the electronic structure at the interface from $a b$ initio DFT cal- 
culations. We focus on the $(\mathrm{LMO})_{1} /(\mathrm{SMO})_{1}$ superlattice and our results suggest that many of the interfacial electronic and magnetic properties shown by this superlattice should also be valid for the more general $(\mathrm{LMO})_{n} /(\mathrm{SMO})_{m}$ superlattices.

We briefly summarize the electronic structure and magnetism for the bulk SMO and LMO compounds. In bulk SMO, the $\mathrm{Mn}$ atoms that are in 4+ charge state have three $d$ electrons which are occupied in the triply degenerate $t_{2 g}$ states. The doubly degenerate $e_{g}$ states, which are higher in energy with respect to $t_{2 g}$ states because of a $\mathrm{MnO}_{6}$ octahedral crystal-field split, remain unoccupied. The $t_{2 g}^{3}$ spin-majority states mediate an antiferromagnetic superexchange to stabilize the G-type antiferromagnetic ordering in the bulk SMO compound, where spin of each Mn atom is opposite to that of the nearest-neighbor $\mathrm{Mn}$ atoms. ${ }^{10,11}$

In bulk LMO the Mn atoms that are in 3+ charge state have four $d$ electrons. Three electrons are occupied in the localized $t_{2 g}$ states and the remaining one electron is occupied in the $e_{g}$ state. The JT distortion to the $\mathrm{MnO}_{6}$ octahedron further splits the $e_{g}$ states into two nondegenerate states: $e_{g}^{1}$ which is lower in energy and $e_{g}^{2}$ which is higher in energy. ${ }^{12}$ The one $e_{g}$ electron is occupied in the $e_{g}^{1}$ state whose lobes are pointed toward the longest $\mathrm{Mn}-\mathrm{O}$ bond. The JT distortion stabilizes the A-type antiferromagnetic structure in the LMO compound. ${ }^{13}$

At the LMO/SMO interface the Mn atoms do not satisfy the $4+$ charge state or the $3+$ charge state to support the bulk magnetism of SMO or LMO. The mixed-valence nature of the $\mathrm{Mn}$ atoms as well as the effect of epitaxial strain create diverse magnetic phases at the interface, which will be analyzed in this section.

Epitaxial strain, arising due to the substrate on which the interface is grown, induces tetragonal distortion to the cubic interface which is quantified by the $c / a$ ratio that differs from one. Experimental studies show different magnetic behaviors at the interface for different $c / a$ ratios. ${ }^{1}$ To obtain the dependence of the magnetic ground state on the strain condition, we have performed total-energy calculations in the range $0.95 \leq c / a \leq 1.05$ for three possible magnetic configurations (A, F, and $\mathrm{C}$ ) (Fig. 1). Magnetic configuration A represents the FM ordering in the $\mathrm{MnO}_{2}$ plane and AFM ordering between the planes. Magnetic configuration $\mathrm{C}$ represents the AFM ordering in the $\mathrm{MnO}_{2}$ plane and FM ordering between the planes and $\mathrm{F}$ represents the FM ordering in all directions. The energetics is shown in Fig. 3 (top).

From the figure we see that for a strong compressive strain (e.g., $c / a=0.95)$, "A" is the most stable magnetic configuration. For the lattice-matched structure $(c / a=1$, no strain), the interface stabilizes with magnetic configuration $\mathrm{F}$ and in case of a strong compressive strain (e.g., $c / a=1.05)$ it stabilizes with magnetic configuration $\mathrm{C}$. The results are in accordance with the experimental observations which show that when the substrates are STO $(c / a=0.98)$, LSAT $(c / a$ $=1.01)$, and $\operatorname{LAO}(c / a=1.05)$, the respective magnetic configurations at the LMO/SMO interface are $\mathrm{A}, \mathrm{F}$, and $\mathrm{C} .{ }^{1}$

We see that as strain changes, the occupancy of the $e_{g}$ orbitals, which controls the magnetic interaction at the interface, also changes. This is shown in Fig. 3 (bottom). For the tensile strain condition $(c / a<1)$ the occupancy of the $x^{2}-y^{2}$ orbital is greater than the occupancy of the $3 z^{2}-1$
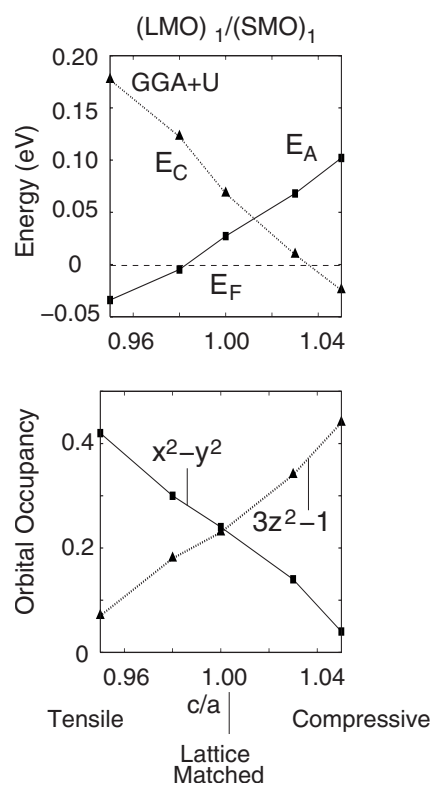

FIG. 3. Total energies for the magnetic configurations, A and C, relative to the energy for configuration $\mathrm{F}$, as a function of the tetragonal distortion $c / a$ (top). The magnetic configurations $\mathrm{A}, \mathrm{F}$, and $\mathrm{C}$ are shown in Fig. 1. Bottom figure shows the occupancies of the $x^{2}-y^{2}$ and the $3 z^{2}-1$ orbitals per $\mathrm{Mn}$ atom as a function of the tetragonal distortion.

orbital and opposite if the strain is compressive $(c / a>1)$. For the lattice-matched structure $(c / a=1)$ both the $e_{g}$ orbitals are more or less equally occupied. Figure 3 also shows that for any value of $c / a$, the nondegenerate $e_{g}$ states combinedly occupy 0.5 electrons which along with three $t_{2 g}$ core electrons make the average valence of the interface $\mathrm{Mn}$ atoms to be +3.5 as expected.

Magnetic interaction between the Mn atoms is determined by the competition between ferromagnetic double exchange ${ }^{14-16}$ via the itinerant $\mathrm{Mn} e_{g}$ electrons and antiferromagnetic superexchange between the localized Mn $t_{2 g}$ core spins. When $x^{2}-y^{2}$ is more occupied and $3 z^{2}-1$ orbital is less occupied (or unoccupied), the strong double exchange in the $\mathrm{MnO}_{2}$ plane strengthens the ferromagnetic ordering while superexchange stabilizes the antiferromagnetic ordering between the planes. The magnetic ordering is opposite to the above when the occupancies of the two $e_{g}$ orbitals are reversed. If both the $e_{g}$ orbitals are more or less equally occupied, the double exchange stabilizes the ferromagnetic ordering both in the plane and between the planes. As described in Secs. III A-III C, a detailed analysis of the density-functional electronic structure of the LMO/SMO interface under different strain conditions gives us a better understanding on the strain induced orbital ordering and its effect on magnetic properties at the interface.

\section{A. $c / a=0.95$, tensile strain}

Tensile strain reduces the out-of-plane lattice parameter $c$ and enhances the in-plane lattice parameter $a$. In other words it decreases the $\mathrm{Mn}-\mathrm{O}$ bond length between the $\mathrm{MnO}_{2}$ planes and increases it in the plane. In such a scenario, the total- 


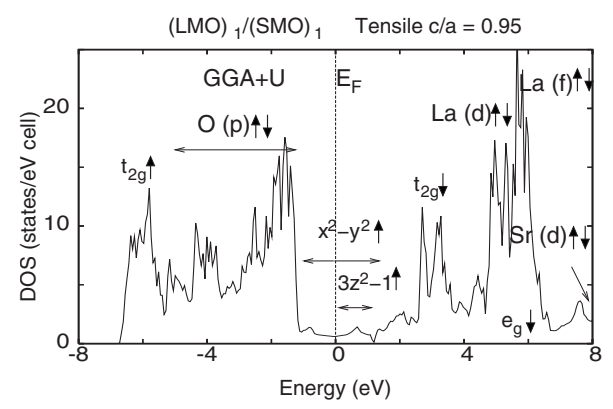

FIG. 4. Total and partial DOSs for the $(\mathrm{LMO})_{1} /(\mathrm{SMO})_{1}$ superlattice $(c / a=0.95)$ in the A-type magnetic configuration. The symbols $\uparrow$ and $\downarrow$ represent the local spin of the atoms. The Mn $e_{g} \uparrow$ state at the Fermi level $\left(E_{F}\right)$ splits into $x^{2}-y^{2}$ and $3 z^{2}-1$ states. The orbital character of the $e_{g}$ states at $E_{F}$ is shown in Fig. 5.

energy calculation (Fig. 3) suggests a stable A-type magnetic configuration (Fig. 1) when tetragonal distortion $(c / a)$ is close to 0.95 . In Fig. 4, we have shown the total and partial densities of states (DOSs) for the $(\mathrm{LMO})_{1} /(\mathrm{SMO})_{1}$ superlattice $(c / a=0.95)$ in the A-type structure obtained from the $\mathrm{GGA}+U$ calculations.

The characteristic features of the electronic structure under tensile strain as seen from Fig. 4 are as follows. The localized Mn $t_{2 g}$ states lie far below the Fermi level $\left(E_{F}\right)$ because of the octahedral crystal field and strong Coulomb repulsion. The $\mathrm{O} p$ states occur in the energy range of -6 to $-1 \mathrm{eV}$. The $x^{2}-y^{2}$ and $3 z^{2}-1$ orbitals are predominant at $E_{F}$. Since the intraplane (on the $x y$-plane) $\mathrm{Mn}-\mathrm{O}$ bond is longer than the interplane (along the z-axis) one (Fig. 2), this lowers the energy of the $x^{2}-y^{2}$ orbital making it more occupied and raises the energy of the $3 z^{2}-1$ orbital, which becomes less occupied.

The origin behind the stability of A-type magnetic configuration for the tensile interface will be explained below. In the bulk LMO, Mn (3+) atom has the electronic configuration $t_{2 g}^{3} e_{g}^{1}$ and in the bulk SMO, Mn (4+) atom has the electronic configuration $t_{2 g}^{3} e_{g}^{0}$. Since at the interface, the $\mathrm{MnO}_{2}$ layers are surrounded by $(\mathrm{SrO})^{0}$ layer and $(\mathrm{LaO})^{1+}$ layer, the interface $\mathrm{Mn}$ atoms are left with the average valence state of +3.5. In such a scenario, the $t_{2 g}$ orbitals will occupy three electrons in the spin-majority states and the $e_{g}$ orbitals will occupy the remaining 0.5 electrons.

Without any occupancy of the $e_{g}$ states, the only contribution to the energy comes from the superexchange interaction between the localized $t_{2 g}$ states to stabilize the G-type AFM phase as in the case of SMO. However, the itinerant $e_{g}$ states, if partially occupied, can mediate the AndersonHasegawa double exchange ${ }^{14}$ to stabilize the FM phase.

The strength of the FM ordering in the plane or out of the plane depends on the occupancy of the individual $x^{2}-y^{2}$ and $3 z^{2}-1$ orbitals. From our calculations (Fig. 3) we find that for tensile strain condition $(c / a=0.95)$, the occupancy of $x^{2}-y^{2}$ orbital is close to 0.45 , while for $3 z^{2}-1$ orbital it is less than 0.1. This is also reflected in the Mn $e_{g}$ band dispersion for the A-type magnetic configuration shown in Fig. 5. Since, the unit cell for the magnetic structure is doubled along the $x y$ plane [i.e., $\left.2 \times(\mathrm{LMO})_{1} /(\mathrm{SMO})_{1}\right]$, for the AFM magnetic configuration we have two $\mathrm{Mn} \uparrow$ and two $\mathrm{Mn} \downarrow$ at-

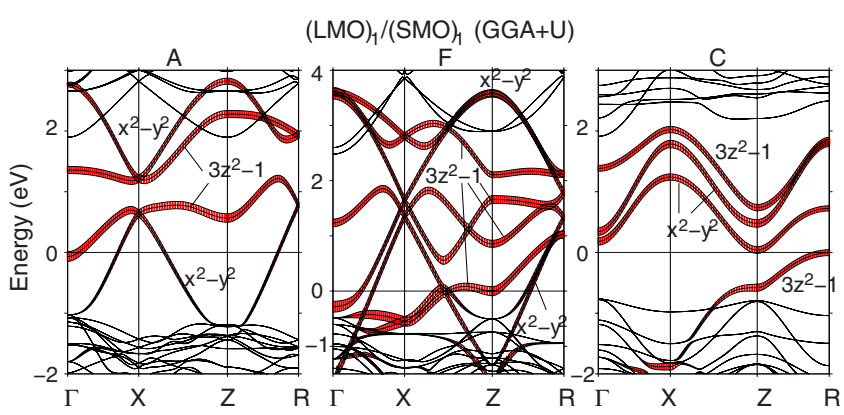

FIG. 5. (Color online) Orbital character of the electron bands near $E_{F}$ for the three magnetic structures A, F, and C. The bands are plotted along the high-symmetry points $\Gamma(0,0,0), X\left(\frac{\pi}{2 a},-\frac{\pi}{2 a}, 0\right)$, $Z\left(0,0,-\frac{\pi}{2 c}\right)$, and $R\left(\frac{\pi}{2 a},-\frac{\pi}{2 a}, \frac{\pi}{2 c}\right)$. The unit cell for the magnetic structures is doubled along the $x y$ plane with the f.u. $2 \times(\mathrm{LMO})_{1} /(\mathrm{SMO})_{1}$. For the AFM configurations (A and C), we have two $M n \uparrow$ and two $M n \downarrow$ atoms. Only spin-up bands are shown for the FM configuration F.

oms. Hence, for the local spin-majority channel, we have two $x^{2}-y^{2}$ orbitals and two $3 z^{2}-1$ orbitals. From the figure we see that the $3 z^{2}-1$ orbitals are mostly in the conduction band and only one $x^{2}-y^{2}$ orbital of two crosses the Fermi level and lies mostly in the valence band. This implies that almost one electron per two Mn atoms in the $x^{2}-y^{2}$ states is occupied which is consistent with the orbital occupancy calculation.

In such a case the $x^{2}-y^{2}$ orbitals will mediate the double exchange mechanism in the $\mathrm{MnO}_{2}$ plane to stabilize a FM ordering in the plane. The gain in kinetic energy due to the planar orbital order, induced by the anisotropic hopping, is more than the loss of superexchange energy. Since the $3 z^{2}-1$ orbitals are only marginally occupied, superexchange between the localized $t_{2 g}$ electrons stabilizes the AFM ordering between the $\mathrm{MnO}_{2}$ planes. The net result is an A-type AFM ordering at the interface.

The valence electron charge-density contours for states in the vicinity of the Fermi level $\left(E_{F}\right)$, shown in Fig. 6, provide a visualization of the above analysis. The charge contours show that the orbital ordering is predominantly $\operatorname{Mn} x^{2}-y^{2}$,

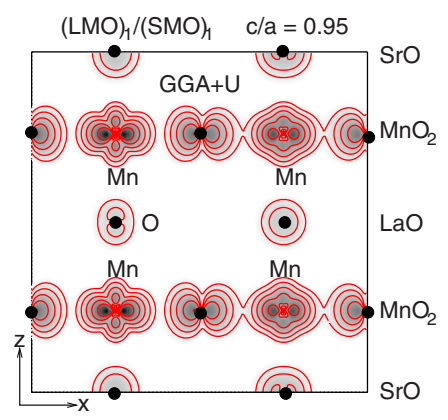

FIG. 6. (Color online) Valence electron charge-density contours plotted on the $x z$ plane in the energy range $E_{F}-0.15 \mathrm{eV}$ to $E_{F}$ to indicate the orbital ordering for the A-type magnetic configuration. Contour values are $\rho_{n}=\rho_{0} \times 10^{n} \delta e / \AA^{3}$, where $\rho_{0}=3.7 \times 10^{-3}$, $\delta=0.4$, and $n$ labels the contours. The charge contours on the $y z$ plane (not shown) are identical to that of $x z$ plane. The orbital ordering is mainly $x^{2}-y^{2}$. 


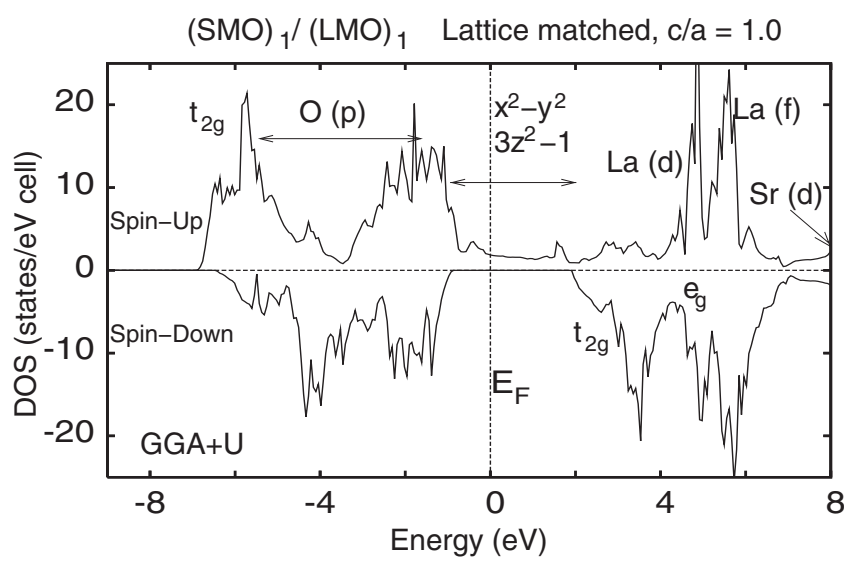

FIG. 7. Total spin-up and spin-down DOSs for $(\mathrm{LMO})_{1} /(\mathrm{SMO})_{1}$ superlattice in the F-type magnetic configuration. Both $x^{2}-y^{2}$ and $3 z^{2}-1$ orbitals are more or less equally occupied. The orbital character of the $e_{g}$ states at $E_{F}$ in the spin-up channel is shown in Fig. 5. The superlattice shows the half-metallic behavior.

$\mathrm{O} p_{x}$, and $p_{y}$, while the occupancies of the $3 z^{2}-1$ and $p_{z}$ orbitals are small. As a result we see a strong coupling between the $\mathrm{Mn} e_{g}$ and $\mathrm{O} p$ orbitals in the plane while it is rather weak between the planes. Therefore, the in-plane magnetic exchange interaction $J^{\prime}$ is ferromagnetic, while the outof-plane $J$ is antiferromagnetic (Fig. 1). Our results are consistent with the experimental results that the magnetic ordering at the interface for $(\mathrm{LMO})_{3} /(\mathrm{SMO})_{2}$ superlattice grown on STO substrate $(c / a=0.98)$ is A type. ${ }^{1}$

\section{B. $c / a=1.0$, lattice-matched structure}

Lattice-matched interfaces are without any tetragonal distortion and hence the in-plane and out-of-plane $\mathrm{Mn}-\mathrm{O}$ bond lengths are identical. Total-energy calculation (Fig. 3) in this case favors a three-dimensional FM ordering ( $\mathrm{F}$ type). To gain insight into the origin behind the FM ground state, we analyze the electronic structure for the lattice-matched interface. In Fig. 7, we have shown the total spin-up and spindown DOSs for the F-type magnetic configuration.

General features of the electronic structure of the latticematched interface are similar to that of the tensile interface. However, now on either side of the Fermi level, both $x^{2}-y^{2}$ and $3 z^{2}-1$ orbitals are predominant in the spin-up channel and they have nearly equal on-site energies. It is due to the fact that the $\mathrm{Mn}-\mathrm{O}$ bond lengths are same both in plane and out of plane, making the $e_{g}$ states nearly degenerate in energy. This is also substantiated from the dispersion of the spin-up Mn $e_{g}$ bands for the F-type structure shown in Fig. 5. Since the f.u. is doubled along the $x y$ plane, there are four $\mathrm{Mn}$ atoms and all are in the same spin orientation. Hence, in the spin-up channel, we have eight $e_{g}$ bands of which almost six lie in the conduction band. Of the remaining two bands, which are part of the valence bands, one is predominantly of $3 z^{2}-1$ character, while the other is predominantly $x^{2}-y^{2}$. Hence the occupancy of each of these orbitals is close to a quarter electron per $\mathrm{Mn}$ atom which is also seen from the orbital occupancy results of Fig. 3. The valence chargedensity contours of Fig. 8 indicate the orbital occupancy of

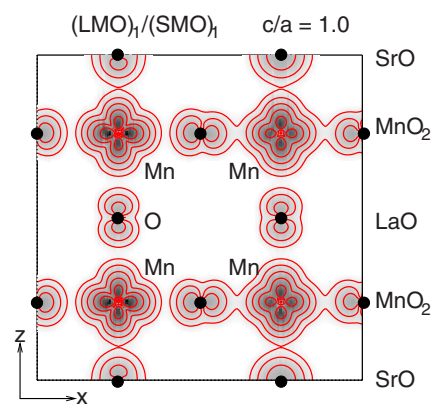

FIG. 8. (Color online) Valence electron charge-density contours plotted on the $x z$ plane in the energy range $E_{F}-0.15 \mathrm{eV}$ to $E_{F}$ to indicate the orbital ordering for the F-type magnetic configuration. Contour values are: $\rho_{n}=\rho_{0} \times 10^{n} \delta_{e} / \AA^{3}$, where $\rho_{0}=3.7 \times 10^{-3}$, $\delta=0.4$, and $n$ labels the contours. The charge contours on the $y z$ plane (not shown) are identical to that of $x z$ plane. The orbital state of each $\mathrm{Mn}$ is a mixture of $x^{2}-y^{2}$ and $3 z^{2}-1$.

the two Mn $e_{g}$ orbitals as well as their hybridization with the $\mathrm{O} p$ orbitals.

The partially occupied $x^{2}-y^{2}$ and $3 z^{2}-1$ orbitals mediate a ferromagnetic double exchange, strong enough to overcome the antiferromagnetic superexchange both in the plane and out of the plane to stabilize a three-dimensional FM ordering. We have shown earlier ${ }^{17}$ that in the case of $\mathrm{CaMnO}_{3} / \mathrm{CaRuO}_{3}$ interface, a leaking of 0.2 electrons from the metallic $\mathrm{CaRuO}_{3}$ side to the $\mathrm{Mn} e_{g}$ states near the interface, which were otherwise unoccupied, is sufficient to stabilize the FM ordering of the Mn spins. In the present case, both the $e_{g}$ orbitals being occupied substantially (more than 0.2 electrons in each orbital), a strong ferromagnetic double exchange coupling along all directions is expected. This is consistent with the experimental observation of ferromagnetism in the LMO/SMO interface structures grown on the LSAT substrate $(c / a=1.01) .{ }^{1}$ The other prominent feature in the electronic structure of the lattice-matched interface is the opening of a gap at the Fermi level in the spin-down channel which makes the system half-metallic (Fig. 7).

\section{C. $c / a=1.05$, compressive strain}

When the strain is compressive, the Mn-O bond length reduces in the $\mathrm{MnO}_{2}$ plane while it increases between the planes. As a result, the $3 z^{2}-1$ orbital is lower in energy and is more occupied, while the $x^{2}-y^{2}$ orbital is higher in energy and is less occupied which is seen from the densities of states (Fig. 9) as well as from the band structure (Fig. 5, right panel).

As in the case of A-type magnetic configuration in the tensile strain condition discussed earlier, here also we have two $\mathrm{Mn} \uparrow$ and two $\mathrm{Mn} \downarrow$ atoms. So for the local spin-majority channel, we have two $x^{2}-y^{2}$ orbitals and two $3 z^{2}-1$ orbitals. From Fig. 5 we see that the $x^{2}-y^{2}$ orbitals lie in the conduction band and only one of the two $3 z^{2}-1$ orbitals lies in the valence band. This shows that the occupancy of the $3 z^{2}-1$ orbital per $\mathrm{Mn}$ atom is close to 0.5 and $x^{2}-y^{2}$ orbitals are basically unoccupied. This is seen from the orbital occupancy (Fig. 3) as well as from the charge-density contour 


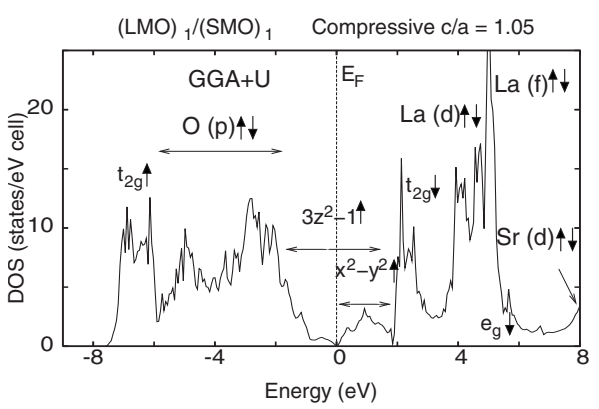

FIG. 9. Total and partial DOSs for the $(\mathrm{LMO})_{1} /(\mathrm{SMO})_{1}$ superlattice with compressive strain $(c / a=1.05)$ in the $\mathrm{C}$-type magnetic configuration. The symbols $\uparrow$ and $\downarrow$ represent the spin-majority and spin-minority states with respect to a $\mathrm{Mn}$ atom. The spin-majority $e_{g}$ band splits into $x^{2}-y^{2}$ (unoccupied) and $3 z^{2}-1$ (partially occupied) bands as seen more clearly from the band-structure plot (Fig. $5)$.

plot of Fig. 10, where we see that the orbital ordering is predominantly $\mathrm{Mn} 3 z^{2}-1$ and $\mathrm{O} p$.

The partially occupied $3 z^{2}-1$ orbital mediates a strong double exchange mechanism to make the out-of-plane magnetic ordering ferromagnetic. The in plane remains antiferomagnetic due to the superexchange between the localized $t_{2 g}$ electrons. Thus the net magnetic configuration for the compressive interface is $\mathrm{C}$ type in agreement with our totalenergy calculations shown in Fig. 3. Experimental studies on $(\mathrm{LMO})_{3} /(\mathrm{SMO})_{2}$ interface, grown on LAO substrate $(c / a=1.05)$, do indeed show a C-type antiferromagnetic configuration $^{1}$ consistent with our theoretical results.

Unlike the lattice-matched interfaces which are metallic, the compressive interface is insulating. It is known that the strong correlation effect in manganites plays an important role to drive the insulating behavior. To elucidate this effect, in Fig. 11 we have plotted the Mn $d$ DOS for different values of $U$. For $U \geq 5 \mathrm{eV}$, a gap opens at the Fermi level to make the interface insulating.

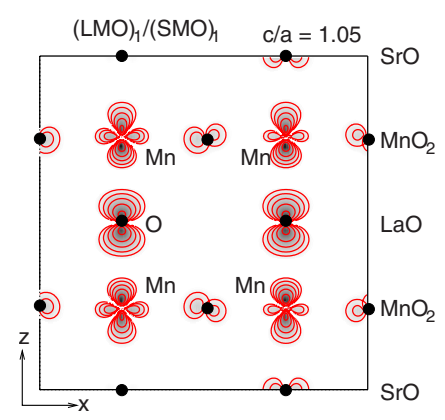

FIG. 10. (Color online) Valence electron charge-density contours plotted on the $x z$ plane in the energy range $E_{F}-0.15 \mathrm{eV}$ to $E_{F}$ indicating the orbital ordering for the C-type magnetic configuration. Contour values are: $\rho_{n}=\rho_{0} \times 10^{n} \delta e / \AA^{3}$, where $\rho_{0}=3.7 \times 10^{-3}$, $\delta=0.4$, and $n$ labels the contours. The charge contours on the $y z$ plane (not shown) are identical to those plotted on the $x z$ plane. The orbital ordering is mainly $3 z^{2}-1$.

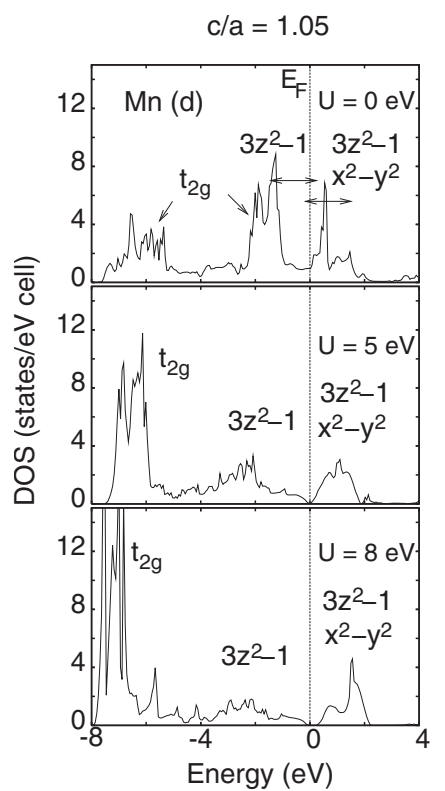

FIG. 11. Atom projected Mn $d$ DOS for a single Mn atom in the spin-majority channel for the $(\mathrm{LMO})_{1} /(\mathrm{SMO})_{1}$ superlattice with the compressive strain condition $(c / a=1.05)$ and the C-type magnetic configuration. The results are obtained from GGA $+U$ with different values of $U$. For $U \geq 5 \mathrm{eV}$ the DOS has a gap at the Fermi level. The $z^{2}-1$ state is split due to the interaction between the Mn atoms on adjacent planes, while the $x^{2}-y^{2}$ state is not because of the AFM alignment of the neighboring $\mathrm{Mn}$ atoms in the plane.

\section{STRAIN EFFECT ON MAGNETISM: A THREE-SITE MODEL}

So far, from the electronic structure calculations for the $(\mathrm{LMO})_{1} /(\mathrm{SMO})_{1}$ superlattice under various strain conditions, we found that strain changes the relative occupancy of the two Mn $e_{g}$ orbitals, which in turn affects the magnetic ordering in the structure. We found that the ordering is antiferromagnetic in the plane and ferromagnetic out of the plane if the strain is compressive and opposite if the strain is tensile, while for the lattice-matched interface, the magnetic ordering is ferromagnetic in all directions. In this section we develop a simple three-site model consisting of the $\mathrm{Mn}$ $\mathrm{O}-\mathrm{Mn}$ atoms to further understand the effect of the straincontrolled orbital occupancy on the magnetic interactions at the interface.

In bulk perovskite manganites, the $t_{2 g}^{3}$ core spins interact via the antiferromagnetic superexchange. In addition to this, the $e_{g}$ electrons mediate the ferromagnetic AndersonHasegawa double exchange ${ }^{14}$ between the core spins, which competes with the antiferromagnetic superexchange. The strength of the double exchange depends on which of the $e_{g}$ orbitals is occupied because of the anisotropic hopping and these are modeled in this section.

In the LMO/SMO superlattice, as seen from the densityfunctional results, the Mn atoms at the interface are left with one extra electron ( 0.5 electrons per $\mathrm{Mn})$ which occupies the itinerant $e_{g}$ states. The epitaxial strain splits the degenerate Mn $e_{g}$ states into $x^{2}-y^{2}$ and $3 z^{2}-1$ states. From the model below, we will see that depending on the strain condition the 


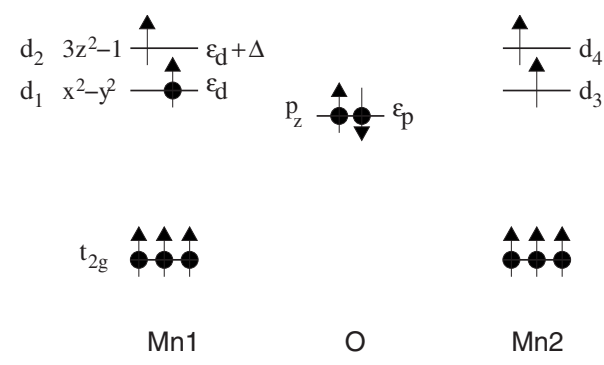

FIG. 12. Orbitals considered in forming the Hamiltonian $H_{\uparrow \uparrow}$ appropriate for the out-of-plane ferromagnetic configuration at the LMO/SMO interface. The parameter $\Delta$ is the on-site energy difference between the $x^{2}-y^{2}$ and $3 z^{2}-1$ orbitals. Depending on the strain condition $\Delta$ can be negative (compressive strain) or positive (tensile strain). The black dots indicate the occupied orbitals for one many-particle configuration, while the arrows indicate the spin states of the orbitals.

relative occupancies of the interface $x^{2}-y^{2}$ and $3 z^{2}-1$ orbitals could switch a ferromagnetic interaction into an antiferromagnetic one and vice versa.

In our model we consider the $t_{2 g}$ electrons as classical core spins which are fixed at the Mn sites with negligible intersite hopping as compared to the itinerant $e_{g}$ electrons, as has been used by many authors in the literature. Hence the Mn-O-Mn double exchange is due to the hopping between the itinerant $x^{2}-y^{2}$ and $3 z^{2}-1$ electrons and $\mathrm{O} p$ electrons (Fig. 12). The model Hamiltonian thus reads

$$
\begin{aligned}
H= & \sum_{i \alpha \sigma} \epsilon_{i \alpha} n_{i \alpha \sigma}+\sum_{\langle i j\rangle \alpha \beta \sigma} t_{i \alpha j \beta}\left(c_{i \alpha \sigma}^{\dagger} c_{j \beta \sigma}+\text { H.c. }\right)+\frac{1}{2} \sum_{i} U_{i} n_{i}\left(n_{i}\right. \\
& -1)-J_{H} \sum_{i \alpha}^{\mathrm{Mn} 1, \mathrm{Mn} 2} \vec{S}_{i} \cdot \vec{S}_{i \alpha}+\frac{1}{2} J_{\mathrm{SX}} \vec{S}_{\mathrm{Mn} 1} \cdot \vec{S}_{\mathrm{Mn} 2}
\end{aligned}
$$

Here, $i, \alpha$, and $\sigma$ are, respectively, the site (Mn or O), orbital $\left(\mathrm{Mn} x^{2}-y^{2}, 3 z^{2}-1, \mathrm{O} p_{x}, p_{y}, p_{z}\right)$, and spin indices. The parameter $\epsilon_{i \alpha}$ is the on-site energy of the orbital, $\langle i j\rangle$ indicates nearest neighbors, $c^{\dagger} s$ are the creation operators, and $n_{i}$ is the total number of electrons at $i$ th site. The matrix elements $t_{i \alpha j \beta}$ are the Slater-Koster tight-binding hopping integrals between the $\mathrm{Mn} e_{g}$ and $\mathrm{O} p$ orbitals. We shall, for simplicity, take the Hun coupling $J_{H}$ as $\infty$ so that only the $e_{g}$ states parallel to the $t_{2 g}$ spin at a Mn site can be occupied. The symbol $\vec{S}_{i}$ represents the $t_{2 g}$ core spins and $\vec{s}_{i \alpha}$ is the spin of the $e_{g}$ electron. The parameter $J_{\mathrm{SX}}$ represents the superexchange between the $t_{2 g}$ core spins. Throughout this paper, we have taken $U_{p}=0$ and $U_{d}=5 \mathrm{eV}$ unless otherwise stated and also the on-site energy of the oxygen orbitals is taken as zero $\epsilon_{p}=0$ for all spins and all three $p$ orbitals.

The net exchange interaction between two $\mathrm{Mn}$ atoms is a sum of the antiferromagnetic superexchange $J_{\mathrm{SX}}$, modeled by the last term in the model Hamiltonian [Eq. (1)], and the double exchange $J_{\mathrm{DX}}$ mediated by the itinerant $e_{g}$ electrons, modeled by the remaining terms in the same equation, so that we have
TABLE I. Slater-Koster tight-binding hopping integrals between the $\mathrm{Mn} e_{g}$ and $\mathrm{O} p$ orbitals. In the three-site model the value of $V_{p d \sigma}$ is taken as $0.9 \mathrm{eV}$.

\begin{tabular}{lcccc}
\hline \hline $\begin{array}{l}\text { Mn } \rightarrow \mathrm{O} \\
\text { direction }\end{array}$ & Orbitals & $\left|p_{x}\right\rangle$ & $\left|p_{y}\right\rangle$ & $\left|p_{z}\right\rangle$ \\
\hline$\hat{x}$ & $\left\langle x^{2}-y^{2}\right|$ & $-\sqrt{3} V_{p d \sigma} / 2$ & 0 & 0 \\
& $\left\langle 3 z^{2}-1\right|$ & $V_{p d \sigma} / 2$ & 0 & 0 \\
$\hat{y}$ & $\left\langle x^{2}-y^{2}\right|$ & 0 & $\sqrt{3} V_{p d \sigma} / 2$ & 0 \\
& $\left\langle 3 z^{2}-1\right|$ & 0 & $V_{p d \sigma} / 2$ & 0 \\
$\hat{z}$ & $\left\langle x^{2}-y^{2}\right|$ & 0 & 0 & 0 \\
& $\left\langle 3 z^{2}-1\right|$ & 0 & 0 & $-V_{p d \sigma}$ \\
\hline \hline & \multicolumn{5}{c}{$J=J_{\mathrm{SX}}+J_{\mathrm{DX}}}$.
\end{tabular}

The exchange interaction $J$ is obtained by calculating the difference between the ground-state energies corresponding to the ferromagnetic (FM) and the antiferromagnetic (AFM) alignments of the two $t_{2 g}$ core spins,

$$
J=E_{\uparrow \uparrow}-E_{\uparrow \downarrow} .
$$

Note that a positive (negative) value of $J$ indicates an AFM (FM) interaction. $J_{\mathrm{SX}}$ is a simple additive term and it is, for the manganites, of the order of $26 \mathrm{meV} .{ }^{18}$ From the present model we will calculate the $J_{\mathrm{DX}}$ which depends on the occupancy of the $e_{g}$ states.

Before we move on to the solution of the model, there is another point that needs to be made. The magnetic interaction between the planes, which is indicated by $J$ in Fig. 1, may differ when we consider the exchange interaction between the Mn spins via the $\mathrm{O} p$ orbitals across the $\mathrm{LaO}$ plane or the SrO plane. However, from the charge contours (e.g., Fig. 6), we see that there is very little difference between the two oxygen atoms located on these planes, so that the Mn$\mathrm{O}-\mathrm{Mn}$ coupling may be expected to be nearly the same. So, in our model, we do not differentiate between these two interactions, so that $J$ is the same across the $\mathrm{LaO}$ plane or the SrO plane. To distinguish the exchange interaction out of the plane and in the plane, we have used the notation $J_{\mathrm{DX}}$ for the former and $\mathrm{J}_{\mathrm{DX}}^{\prime}$ for the latter in the remaining part of this section.

\section{A. Out-of-plane exchange $J$}

First consider the out-of-plane exchange by evaluating the ground-state energies for the ferromagnetic and the antiferromagnetic configurations of the $\mathrm{Mn} t_{2 g}$ spins of two Mn atoms along the $z$ axis. The model for the out-of-plane FM configuration is schematically shown in Fig. 12. Listed in Table I are the Slater-Koster tight-binding hopping integrals between $\mathrm{Mn} e_{g}$ and $\mathrm{O} p$ orbitals, which shows that out of the three $\mathrm{O} p$ orbitals, only $p_{z}$ takes part in the hopping process along the $z$ axis. Therefore, in this model we have five spin-up orbitals $\left(\mathrm{O} p_{z}\right.$, two $\mathrm{Mn} z^{2}-1$, and two $\left.\mathrm{Mn} x^{2}-y^{2}\right)$ available for two spin-up electrons and one spin-down orbital $\left(\mathrm{O} p_{z}\right)$ available for the lone spin-down electron. The 
spin-down electron can only be on the $\mathrm{O} p_{z}$ orbital in the ferromagnetic case due to the infinite $J_{H}$ and does not take part in the hopping process. Hence, we have a tendimensional two-particle configuration space $\left({ }^{5} C_{2} \times{ }^{1} C_{1}\right)$.

We choose the two-particle (both particles with spins up) basis set in the order $\left|p d_{1}\right\rangle,\left|d_{1} d_{2}\right\rangle,\left|d_{1} d_{4}\right\rangle,\left|p d_{2}\right\rangle,\left|p d_{4}\right\rangle,\left|d_{2} d_{4}\right\rangle$, $\left|p d_{3}\right\rangle,\left|d_{2} d_{3}\right\rangle,\left|d_{3} d_{4}\right\rangle$, and $\left|d_{1} d_{3}\right\rangle$, where $p, d_{1}, d_{2}, d_{3}$, and $d_{4}$, respectively, denote the $\mathrm{O} p_{z}, \mathrm{Mn} 1 x^{2}-y^{2}, \mathrm{Mn} 13 z^{2}-1$, Mn $2 x^{2}-y^{2}$, and Mn2 $3 z^{2}-1$ orbitals. With this basis set, for the case of the two Mn $t_{2 g}$ core spins ferromagnetically aligned, the Hamiltonian for the itinerant electrons $\mathrm{H}_{\uparrow \uparrow}$ becomes

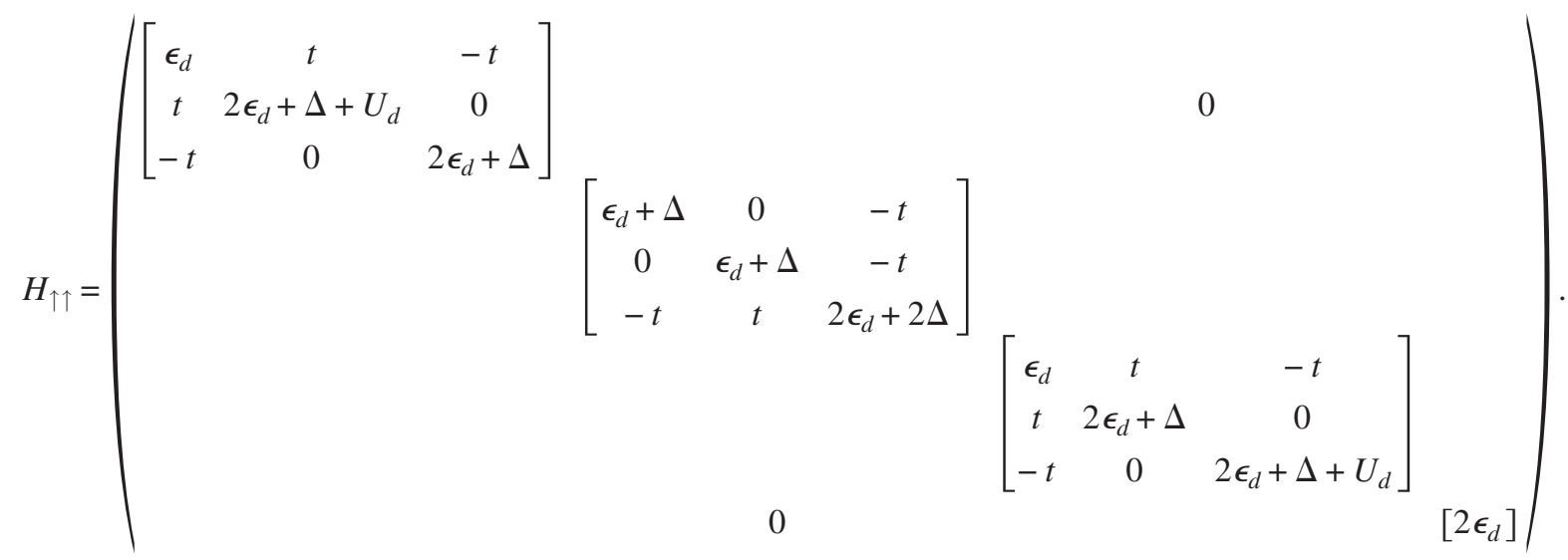

Again, here $\epsilon_{d}$ represents the on-site energy for the Mn $x^{2}-y^{2}$ orbitals and $\Delta$ is the energy shift of the Mn $z^{2}-1$ orbital from the $x^{2}-y^{2}$ orbital due to strain. The on-site energy of the $\mathrm{O} p$ orbitals is taken as zero. Parameter $t$ is the hopping matrix element $\left(V_{p d \sigma}\right)$. From DFT calculations, we found that for compressive strain condition $\Delta$ is +ve and for tensile strain condition $\Delta$ is -ve. For the lattice-matched interface the on-site energies of the $x^{2}-y^{2}$ and the $3 z^{2}-1$ orbitals are about the same.

We now consider the antiferromagnetic case, where the two Mn $t_{2 g}$ spins are aligned antiferromagnetically. In this case, as seen from Fig. 13, we see that there are six active orbitals, three spin-up and three spin-down. One can populate these orbitals with two spin-up electrons and one spin-down electrons or vice versa. (We do not consider the configurations where all three electrons have the same spins as this would correspond to an oxygen-to-manganese charge-transfer state, which has a much higher energy.) In either of these cases we have a nine-dimensional three particle configuration space $\left({ }^{3} C_{2} \times{ }^{3} C_{1}\right)$. These two sets of configurations do not interact with each other, as the model Hamiltonian [Eq. (1)] does not allow hopping between two opposite spins and have the same ground-state energy.

By considering the configurations with two spin-up electrons and one spin-down electron and arranging the basis set in the order $\left|p \bar{p} d_{1}\right\rangle,\left|\bar{p} d_{1} d_{2}\right\rangle,\left|p d_{1} \bar{d}_{4}\right\rangle,\left|d_{1} d_{2} \bar{d}_{4}\right\rangle,\left|p d_{1} \bar{d}_{3}\right\rangle,\left|d_{1} d_{2} \bar{d}_{3}\right\rangle,\left|p \bar{p} d_{2}\right\rangle,\left|p d_{2} \bar{d}_{4}\right\rangle$, and $\left|p d_{2} \bar{d}_{3}\right\rangle$, where the bar stands for the spin-down orbitals and unbar stands for the spin-up orbitals, the antiferromagnetic Hamiltonian, $H_{\uparrow \downarrow}$, becomes

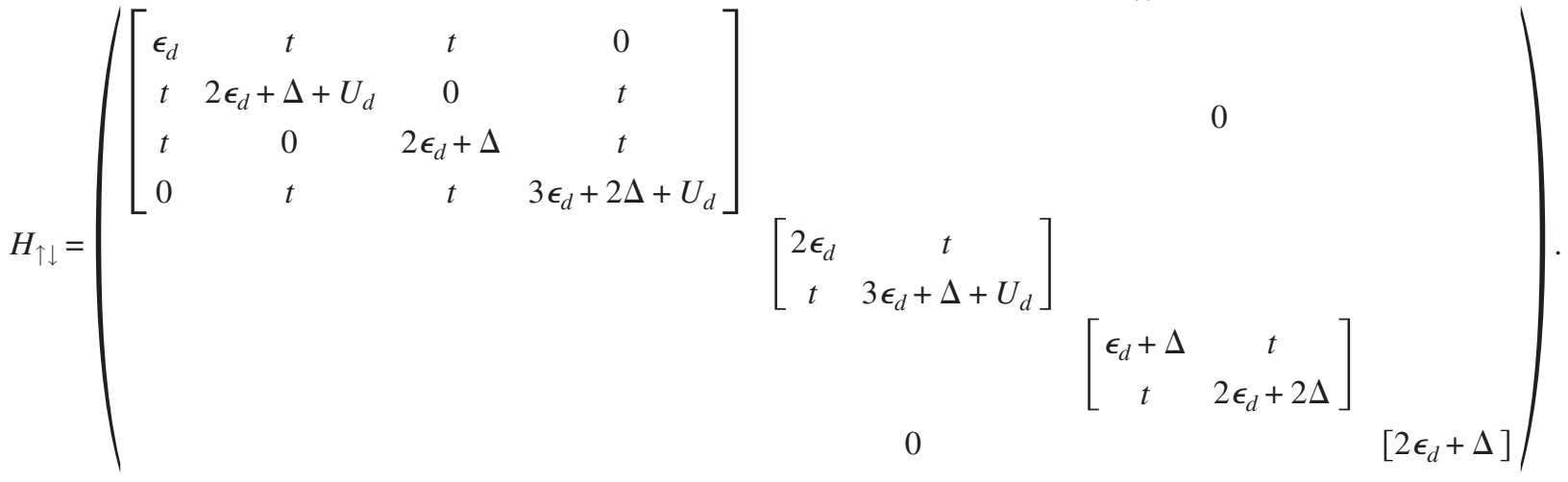

The above matrices can be diagonalized easily for all parameter values; however, for some limiting cases, one can solve these either analytically or using the perturbation theory, which then gives us considerable insights into the resulting exchange interactions.
To this end, we first consider the limit when $\Delta$ is sufficiently large and negative. One immediately sees by inspecting the matrix that the ground state of $H_{\uparrow \uparrow}$ comes from the second block diagonal of Eq. (4). Diagonalization then yields the ground-state energy to be 


$$
E_{\uparrow \uparrow}=3\left(\epsilon_{d}+\Delta\right) / 2-\sqrt{\left(\epsilon_{d}+\Delta\right)^{2}+8 t^{2}} / 2 .
$$

For the AFM Hamiltonian $H_{\uparrow \downarrow}$ in the same limit for $\Delta$, the ground state comes from the third block diagonal of Eq. (5), yielding immediately the ground-state energy,

$$
E_{\uparrow \downarrow}=3\left(\epsilon_{d}+\Delta\right) / 2-\sqrt{\left(\epsilon_{d}+\Delta\right)^{2}+4 t^{2}} / 2 .
$$

The out-of-plane exchange energy $J_{\mathrm{DX}}$ is obtained by taking the difference between the FM and AFM ground-state energies, so that for the case of the large and negative values of $\Delta$ that we are considering, we get

$$
J_{\mathrm{DX}}=E_{\uparrow \uparrow}-E_{\uparrow \downarrow}=1 / 2 \times\left(\sqrt{\left(\epsilon_{d}+\Delta\right)^{2}+4 t^{2}}-\sqrt{\left(\epsilon_{d}+\Delta\right)^{2}+8 t^{2}}\right) .
$$

As seen from Eq. (8), the exchange energy is negative irrespective of the Hamiltonian parameters, indicating that the out-of-plane double exchange is ferromagnetic and robust. We have to add the superexchange term $J_{\mathrm{SX}}$ to this to get the net magnetic interaction. The results are consistent with the numerically computed value of $J$ presented in Fig. 14, which will be discussed later.

Similarly, we can also obtain an expression for a large and positive $\Delta$. Again, it is immediately clear from the inspection of the Hamiltonians [Eqs. (4) and (5)] that the FM and AFM ground states come from the first sub-block of $H_{\uparrow \uparrow}$ and $H_{\uparrow \downarrow}$. An analytical diagonalization is not possible in this case, however one can apply the fourth-order nondegenerate perturbation theory to compute $E_{\uparrow \uparrow}$ and $E_{\uparrow \downarrow}$, the difference of which yields the result

$$
J_{\mathrm{DX}}=\frac{t^{4}}{2 \epsilon_{d}+2 \Delta+U_{d}}\left(\frac{1}{\epsilon_{d}+\Delta+U_{d}}+\frac{1}{\epsilon_{d}+\Delta}\right)^{2}
$$

We see that if the $x^{2}-y^{2}$ orbital is more occupied (i.e., $\Delta$ $>0$ ), the out-of-plane magnetic ordering is AFM for any values of $\epsilon_{d}$ and $\Delta$.

In Fig. 14, we have calculated the exchange interaction by simply a numerical diagonalization of the two Hamiltonian matrices for a general value of $\Delta$, viz., $-1.0 \mathrm{eV} \leq \Delta$ $\leq 1.0 \mathrm{eV}$. The numerical results for $J$ are consistent with
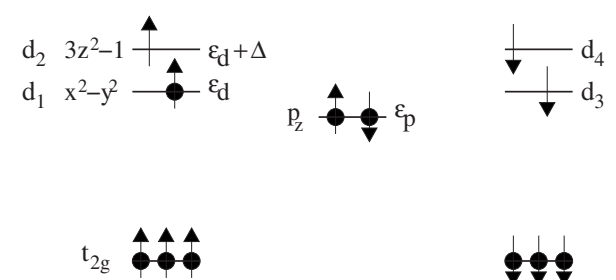

$\mathrm{Mn} 1$

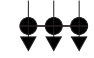

$\mathrm{Mn} 2$
FIG. 13. Orbitals considered in forming the Hamiltonian $H_{\uparrow \downarrow}$ appropriate for the out-of-plane exchange. Of the three oxygen $p$ orbitals, only $p_{z}$ has a nonzero hopping to the $\mathrm{Mn} e_{g}$ orbitals. our analytical and perturbation results for the large values of $\Delta$. When $\Delta$ is less than $-0.15 \mathrm{eV}$ we have a FM interaction and if it is greater than $0.15 \mathrm{eV}$ we have an AFM interaction. For the intermediate region of $\Delta(-0.15 \mathrm{eV} \leq \Delta$ $\leq 0.15 \mathrm{eV}$ ), the ground state swiftly changes between FM and AFM.

Our model is consistent with the DFT calculations discussed in Sec. IV A (Fig. 3), where we see that for compressive strain the $3 z^{2}-1$ orbitals are more occupied $(\Delta<0)$ and the total-energy calculation yields an out-of-plane FM configuration. If the strain is tensile, the $x^{2}-y^{2}$ orbital is more occupied $(\Delta>0)$ and the out-of-plane magnetic ordering is AFM.

\section{B. In-plane exchange $J^{\prime}$}

Now we consider the Mn-O-Mn exchange in the plane (xy plane), i.e., between two $\mathrm{Mn}$ atoms on a $\mathrm{MnO}_{2}$ plane adjacent to the interface. In this case $p_{x}, p_{y}$, and $e_{g}$ are the only active orbitals since the hopping between $p_{z}$ and $e_{g}$ orbitals is not allowed (Table I). For concreteness we have taken the Mn-O-Mn to be along the $x$ axis and results are identical if it is along the $y$ axis.

First consider the FM configuration of the two Mn $t_{2 g}$ spins. Here the itinerant electrons have a ten-dimensional configuration space as in the case of out of plane but with $p_{x}$ as the active orbital instead of $p_{z}$. Taking the two-particle basis set in the order $\left|p d_{1}\right\rangle,\left|p d_{2}\right\rangle,\left|p d_{3}\right\rangle,\left|p d_{4}\right\rangle,\left|d_{1} d_{2}\right\rangle,\left|d_{1} d_{3}\right\rangle$, $\left|d_{1} d_{4}\right\rangle,\left|d_{2} d_{3}\right\rangle,\left|d_{2} d_{4}\right\rangle$, and $\left|d_{3} d_{4}\right\rangle$ and denoting by $t^{\prime}$ and $t^{\prime \prime}$ the hopping matrix elements $\frac{V_{p d \sigma}}{2}$ and $\frac{\sqrt{3} V_{p d \sigma}}{2}$, respectively, the Hamiltonian $H_{\uparrow \uparrow}$ becomes

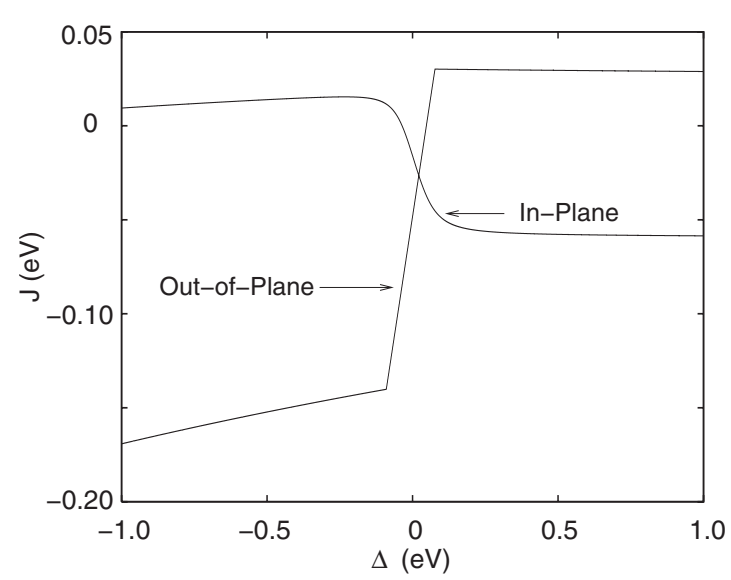

FIG. 14. Exchange parameter $J\left(=J_{\mathrm{DX}}+J_{\mathrm{SX}}\right)$, with $J_{\mathrm{DX}}$ obtained from the numerical diagonalization of the Hamiltonians [Eq. (4), (5), (10), and (11)], as a function of energy difference $\Delta$ between the $x^{2}-y^{2}$ and the $3 z^{2}-1$ orbitals. For the calculation of $J$, the parameters $\epsilon_{d}, U_{d}, V_{p d \sigma}$, and $J_{\mathrm{SX}}$ are taken as $5 \mathrm{eV}, 5 \mathrm{eV}, 0.9 \mathrm{eV}$, and $26 \mathrm{meV}$ respectively. When $\Delta$ is sufficiently positive (tensile strain) the magnetic interaction is FM in the plane $\left(J^{\prime}\right)$ and AFM between the planes $(J)$. When $\Delta$ is sufficiently negative (compressive strain) the magnetic interaction reverses. 


$$
H_{\uparrow \uparrow}=\left(\begin{array}{cccccccccc}
\epsilon_{d} & 0 & 0 & 0 & -t^{\prime} & -t^{\prime \prime} & t^{\prime} & 0 & 0 & 0 \\
0 & \epsilon_{d}+\Delta & 0 & 0 & -t^{\prime \prime} & 0 & 0 & -t^{\prime \prime} & t^{\prime} & 0 \\
0 & 0 & \epsilon_{d} & 0 & 0 & -t^{\prime \prime} & 0 & t^{\prime} & 0 & t^{\prime} \\
0 & 0 & 0 & \epsilon_{d}+\Delta & 0 & 0 & -t^{\prime \prime} & 0 & t^{\prime} & t^{\prime \prime} \\
-t^{\prime} & -t^{\prime \prime} & 0 & 0 & 2 \epsilon_{d}+\Delta+U_{d} & 0 & 0 & 0 & 0 & 0 \\
-t^{\prime \prime} & 0 & -t^{\prime \prime} & 0 & 0 & 2 \epsilon_{d} & 0 & 0 & 0 & 0 \\
t^{\prime} & 0 & 0 & -t^{\prime \prime} & 0 & 0 & 2 \epsilon_{d}+\Delta & 0 & 0 & 0 \\
0 & -t^{\prime \prime} & t^{\prime} & 0 & 0 & 0 & 0 & 2 \epsilon_{d}+\Delta & 0 & 0 \\
0 & t^{\prime} & 0 & t^{\prime} & 0 & 0 & 0 & 0 & 2 \epsilon_{d}+2 \Delta & 0 \\
0 & 0 & t^{\prime} & t^{\prime \prime} & 0 & 0 & 0 & 0 & 0 & 2 \epsilon_{d}+\Delta+U_{d}
\end{array}\right) .
$$

Similar to out-of-plane AFM Hamiltonian, the in-plane AFM Hamiltonian has a nine-dimensional configuration space. If we choose the basis set in the order $\left|p \bar{p} d_{1}\right\rangle,\left|p \bar{p} d_{2}\right\rangle,\left|\bar{p} d_{1} d_{2}\right\rangle,\left|p d_{1} \bar{d}_{3}\right\rangle,\left|p d_{2} \bar{d}_{3}\right\rangle,\left|d_{1} d_{2} \bar{d}_{3}\right\rangle,\left|p d_{1} \bar{d}_{4}\right\rangle,\left|p d_{2} \bar{d}_{4}\right\rangle$, and $\left|d_{1} d_{2} \bar{d}_{4}\right\rangle$, the antiferromagnetic Hamiltonian $H_{\uparrow \downarrow}$ for the present case reads

$$
H_{\uparrow \downarrow}=\left(\begin{array}{ccccccccc}
\epsilon_{d} & 0 & -t^{\prime} & t^{\prime \prime} & 0 & 0 & -t^{\prime} & 0 & 0 \\
0 & \epsilon_{d}+\Delta & -t^{\prime \prime} & 0 & t^{\prime \prime} & 0 & 0 & -t^{\prime} & 0 \\
-t^{\prime} & -t^{\prime \prime} & 2 \epsilon_{d}+\Delta+U_{d} & 0 & 0 & t^{\prime \prime} & 0 & 0 & -t^{\prime} \\
t^{\prime \prime} & 0 & 0 & 2 \epsilon_{d} & 0 & -t^{\prime} & 0 & 0 & 0 \\
0 & t^{\prime \prime} & 0 & 0 & 2 \epsilon_{d}+\Delta & -t^{\prime \prime} & 0 & 0 & 0 \\
0 & 0 & t^{\prime \prime} & -t^{\prime} & -t^{\prime \prime} & 3 \epsilon_{d}+\Delta+U_{d} & 0 & 0 & 0 \\
-t^{\prime} & 0 & 0 & 0 & 0 & 0 & 2 \epsilon_{d}+\Delta & 0 & -t^{\prime} \\
0 & -t^{\prime} & 0 & 0 & 0 & 0 & 0 & 2 \epsilon_{d}+2 \Delta & -t^{\prime \prime} \\
0 & 0 & -t^{\prime} & 0 & 0 & 0 & -t^{\prime} & -t^{\prime \prime} & 3 \epsilon_{d}+2 \Delta+U_{d}
\end{array}\right) .
$$

An analytical diagonalization for both the ferromagnetic and antiferromagnetic Hamiltonians is not possible due to their non-block-diagonal nature and large dimension. Nondegenerate perturbation theory cannot be applied as $H_{\uparrow \uparrow}$ contains degenerate states. However, in the limit $\Delta \rightarrow \infty$ the ground-state energies for the FM and AFM configurations are obtained from a $3 \times 3$ submatrix of $H_{\uparrow \uparrow}$ and a $2 \times 2$ submatrix of $H_{\uparrow \downarrow}$, respectively. The in-plane exchange energy $J_{\mathrm{DX}}^{\prime}$ in the limit $\Delta \rightarrow \infty$ is then immediately obtained as

$$
J_{\mathrm{DX}}^{\prime}=\left(\sqrt{\epsilon_{d}^{2}+4 t^{\prime \prime 2}}-\sqrt{\epsilon_{d}^{2}+8 t^{\prime \prime 2}}\right) / 2 .
$$

Here, we see that for large and positive value of $\Delta, J_{\mathrm{DX}}$ is negative quantity which implies a FM interaction in the plane.

Similarly, in the limit $\Delta \rightarrow-\infty$, we find that

$$
J_{\mathrm{DX}}^{\prime}=\left[\epsilon_{d}+U_{d}+\sqrt{\left(\epsilon_{d}+U_{d}\right)^{2}+4 t^{\prime \prime 2}}\right] / 2 .
$$

Equation (13) shows that for large and negative value of $\Delta$, we have an AFM configuration in the plane.

In Fig. 14 we have plotted the in-plane exchange $J^{\prime}$ as a function of $\Delta$ obtained from direct numerical diagonalization of the full Hamiltonians $H_{\uparrow \uparrow}$ and $H_{\uparrow \downarrow}$, which indeed shows that for the in-plane exchange $J^{\prime}$, the magnetic interaction in the plane switches from ferromagnetic to antiferromagnetic, as $\Delta$ is changed from positive to negative values.
To summarize our analysis in this section, we find that the strain-induced splitting in the $e_{g}$ states is instrumental in determining the magnetic properties at the interface. If $x^{2}-y^{2}$ orbital is relatively more occupied than the $3 z^{2}-1$ orbital, the magnetic ordering at the $\mathrm{LMO} / \mathrm{SMO}$ interface is more likely to be A type with the FM and AFM configurations stabilized in the pane and out of the plane, respectively. If the $3 z^{2}-1$ orbital is more occupied the magnetic ordering is more likely to be $\mathrm{C}$ type with in-plane AFM ordering and out-of-plane FM ordering. When both the $e_{g}$ orbitals are more or less equally occupied, the double exchange wins over the superexchange to stabilize the interface in a three-dimensional FM configuration. The exchange interactions calculated from the three-site model are in good agreement with the results obtained from DFT calculations as shown in Table II.

\section{EFFECT OF STRAIN ON A THICKER SUPERLATTICE: $\left(\mathrm{LaMnO}_{3}\right)_{1} /\left(\mathrm{SrMnO}_{3}\right)_{3}$}

In Secs. I-IV we have studied the effects of strain on the magnetic interactions in the $(\mathrm{LMO})_{1} /(\mathrm{SMO})_{1}$ superlattice. To generalize the strain effects on magnetism at the interface, in this section we have analyzed the magnetic properties of a thicker superlattice, viz., $(\mathrm{LMO})_{1} /(\mathrm{SMO})_{3}$, which has both inner and interfacial $\mathrm{MnO}_{2}$ planes.

In the $(\mathrm{LMO})_{1} /(\mathrm{SMO})_{3}$ superlattice, we have taken the G-type AFM configuration for the inner $\mathrm{MnO}_{2}$ planes as they 
TABLE II. Mn-O-Mn exchange energy (in meV) in the plane $\left(J^{\prime}\right)$ and out of the plane $(J)$ obtained from the DFT calculations and the three-site model for different values of the $c / a$ ratio. The model uses the $\Delta$ values corresponding roughly to the DFT results for the three different strain conditions and the other parameters are: $\epsilon_{d}=U_{d}=5 \mathrm{eV}, V_{p d \sigma}=0.9 \mathrm{eV}$, and $J_{\mathrm{SX}}=26 \mathrm{meV}$ (Ref. 18).

\begin{tabular}{rrrrrrr}
\hline \hline & \multicolumn{2}{c}{ DFT } & & \multicolumn{3}{c}{ Model } \\
\cline { 2 - 3 } \cline { 5 - 7 }$c / a$ & \multicolumn{1}{c}{$J^{\prime}$} & $J$ & & $\Delta(\mathrm{eV})$ & $J^{\prime}$ & \multicolumn{1}{c}{$J$} \\
\hline 0.95 & -85 & 34 & & 1.0 & -58 & 29 \\
1.00 & -27 & -30 & & 0.0 & -18 & -44 \\
1.05 & 14 & -100 & & -1.0 & 11 & -164 \\
\hline \hline
\end{tabular}

belong to the SMO constituent of the superlattice. For the interfacial $\mathrm{MnO}_{2}$ planes, we have considered again the F-type, A-type, and C-type magnetic configurations (Fig. 1). In Fig. 15, we have shown the energetics for these three magnetic configurations as a function of the in-plane lattice parameter $a$. From the figure we see that for lower values of the lattice constant $a$, the interface shows a stable C-type magnetic ordering and as we increase the value of $a$, the interface gradually moves toward an F-type magnetic configuration. For very high values of $a$, the interface stabilizes with the A-type magnetic configuration.

For a strain free LMO/SMO superlattice, the in-plane lattice parameter coincides with the average lattice parameter $a_{0}^{1}$ (see Sec. II), which is $3.835 \AA$ for the $(\mathrm{LMO})_{1} /(\mathrm{SMO})_{3}$ superlattice. As in the case of $(\mathrm{LMO})_{1} /(\mathrm{SMO})_{1}$ superlattice (Fig. 3), here also we see that for strong compressive strain condition, the stable magnetic ordering at the interface is $\mathrm{C}$ type, while for strong tensile strain condition the magnetic ordering is $\mathrm{F}$ type. For the lattice-matched interface we see a stable FM ordering (F type) in all directions. Our bandstructure calculation shows insulating behavior for strong compressive strain condition, while for lattice-matched and tensile strain conditions it is metallic.

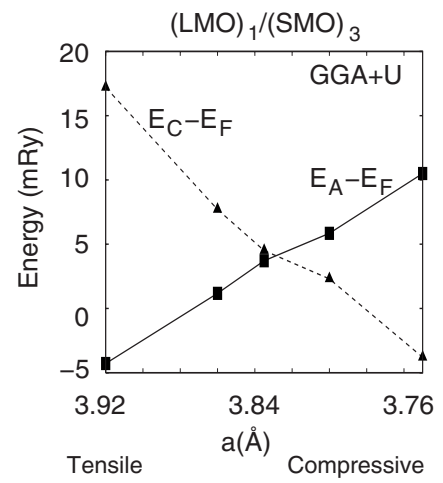

FIG. 15. Total energies for magnetic configurations $\mathrm{A}$ and $\mathrm{C}$ relative to the energy for the magnetic configuration $\mathrm{F}$ as a function of the in-plane lattice parameter $a$ for the $(\mathrm{LMO})_{1} /(\mathrm{SMO})_{3}$ superlattice. The magnetic configurations A, F, and $\mathrm{C}$ are shown in Fig. 1. For strong tensile strain condition, we see a stable A-type magnetic ordering for the interfacial $\mathrm{MnO}_{2}$ layers, while for strong compressive strain condition the magnetic ordering is $\mathrm{C}$ type. For the latticematched case, the ferromagnetic ordering is stabilized in all directions.

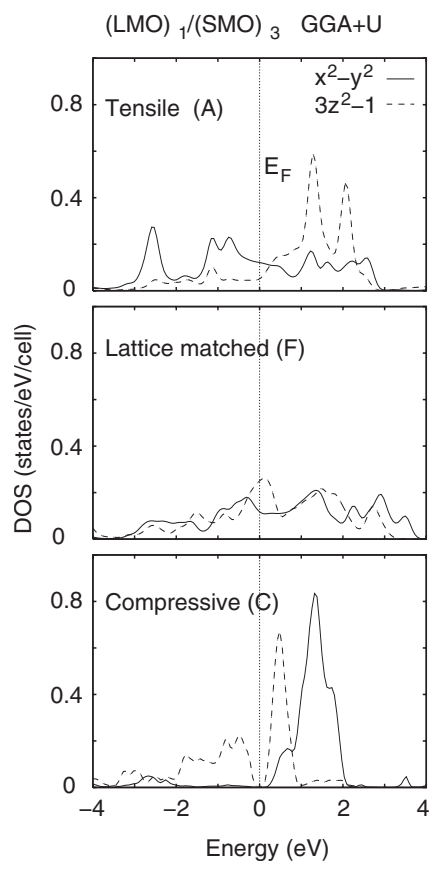

FIG. 16. Spin-majority Mn $e_{g}$ DOS corresponding to the interface $\mathrm{Mn}$ atoms for the $(\mathrm{LMO})_{1} /(\mathrm{SMO})_{3}$ superlattice. The results are shown for the in-plane lattice parameter $a=3.92 \AA$ (tensile), $3.835 \AA$ (lattice matched), and $3.765 \AA$ (compressive). If the strain condition is tensile, the $x^{2}-y^{2}$ orbital is more occupied and the $3 z^{2}-1$ orbital is less occupied and opposite if the strain condition is compressive. For the lattice-matched condition, both $x^{2}-y^{2}$ and $3 z^{2}-1$ orbitals are more or less equally occupied.

To study the role of Mn $e_{g}$ orbitals on the interfacial magnetism in $(\mathrm{LMO})_{1} /(\mathrm{SMO})_{3}$ superlattice, in Fig. 16 we have shown the orbital projected Mn $e_{g}$ DOS for the interface Mn atoms for $a=3.92 \AA$ (tensile strain), $3.835 \AA$ (lattice matched), and $3.75 \AA$ (compressive strain). From the figure we see that, analogous to the case of $(\mathrm{LMO})_{1} /(\mathrm{SMO})_{1}$ superlattice, for the tensile strain condition the $x^{2}-y^{2}$ is relatively more occupied and $3 z^{2}-1$ orbital is less occupied and opposite for the compressive strain condition. For the latticematched interface, both the $x^{2}-y^{2}$ and $3 z^{2}-1$ orbitals are more or less equally occupied as before.

Hence, as discussed earlier, for the tensile strain condition, we have a strong ferromagnetic double exchange coupling in the $\mathrm{MnO}_{2}$ plane through $x^{2}-y^{2}$ orbitals while between the planes we have an antiferromagnetic coupling due to superexchange between the $t_{2 g}$ core spins. For the compressive strain condition the higher occupancy of Mn $3 z^{2}-1$ orbitals leads to a ferromagnetic coupling between the planes and due to negligible occupancy of the $x^{2}-y^{2}$ orbital, the antiferromagnetic ordering is sustained in the $\mathrm{MnO}_{2}$ plane. For the lattice-matched structure, the orbital ordering is a combination of both $x^{2}-y^{2}$ and $3 z^{2}-1$ orbitals and hence strong double exchange coupling both in the plane and out of the plane stabilizes the ferromagnetic interaction in all directions. The similarity in the interfacial magnetic properties for both the superlattices, viz., $(\mathrm{LMO})_{1} /(\mathrm{SMO})_{1}$ and $(\mathrm{LMO})_{1} /(\mathrm{SMO})_{3}$, for different strain conditions suggests that the strain effect on magnetism at the LMO/SMO interface may be true for the general $(\mathrm{LMO})_{n} /(\mathrm{SMO})_{m}$ superlattice as well. 


\section{SUMMARY}

In summary, we have studied the effect of the epitaxial strain on the magnetic ordering at the interface of LMO/ SMO superlattices by a detailed analysis on the $(\mathrm{LMO})_{1} /(\mathrm{SMO})_{1}$ superlattice. We found that the epitaxial strain induces different orbital orderings which in turn change the magnetic ordering at the interface. The magnetic ordering at the interface is determined by the competition between the antiferromagnetic superexchange between the core $t_{2 g}$ electrons and ferromagnetic double exchange between the itinerant $e_{g}$ electrons. The strength of the latter in the $\mathrm{MnO}_{2}$ plane or between the planes strongly depends on the occupancy of the nondegenerate $e_{g}$ orbitals.

In case of a strong tensile strain condition, the higher occupancy of the $x^{2}-y^{2}$ orbital strengthens the double exchange coupling to stabilize the ferromagnetic ordering in the $\mathrm{MnO}_{2}$ plane, while between the $\mathrm{MnO}_{2}$ planes the reduced double exchange coupling, due to the negligibly occupied $3 z^{2}-1$ orbital, fails to overcome the antiferromagnetic $t_{2 g}-t_{2 g}$ superexchange and stabilizes the A-type magnetic ordering at the interface. For strong compressive strain condition, the magnetic ordering reverses, viz., higher occupancy of the
$3 z^{2}-1$ orbital lead to a ferromagnetic coupling between the $\mathrm{MnO}_{2}$ planes, while the depleted $x^{2}-y^{2}$ occupancy allows the antiferromagnetic ordering in the plane to make the C-type magnetic configuration as the most stable one. For a lattice-matched structure, double exchange is strong enough both in the $\mathrm{MnO}_{2}$ plane and between the planes, due to more or less equally occupied $x^{2}-y^{2}$ and $3 z^{2}-1$ orbitals, to allow ferromagnetic ordering in all directions.

The electronic structure calculations for the $(\mathrm{LMO})_{1} /(\mathrm{SMO})_{3}$ superlattice showed that the epitaxial strain affects the magnetism at the interface in a similar way as the $(\mathrm{LMO})_{1} /(\mathrm{SMO})_{1}$ superlattice. This suggests that the strain effect on magnetism may be similar for the general $(\mathrm{LMO})_{n} /(\mathrm{SMO})_{m}$ superlattice. In addition, similar considerations regarding the effects of strain on orbital ordering and magnetism should be valid for interfaces between other perovskite oxides as well.

\section{ACKNOWLEDGMENT}

This work was supported by the U.S. Department of Energy under Grant No. DE-FG02-00ER45818.
${ }^{1}$ H. Yamada, M. Kawasaki, T. Lottermoser, T. Arima, and Y. Tokura, Appl. Phys. Lett. 89, 052506 (2006)

${ }^{2}$ T. Koida, M. Lippmaa, T. Fukumura, K. Itaka, Y. Matsumoto, M. Kawasaki, and H. Koinuma, Phys. Rev. B 66, 144418 (2002).

${ }^{3}$ P. A. Salvador, A.-M. Haghiri-Gosnet, B. Mercey, M. Hervieu, and B. Raveau, Appl. Phys. Lett. 75, 2638 (1999).

${ }^{4}$ A. Bhattacharya, X. Zhai, M. Warusawithana, J. N. Eckstein, and S. D. Bader, Appl. Phys. Lett. 90, 222503 (2007).

${ }^{5}$ S. Smadici, P. Abbamonte, A. Bhattacharya, X. Zhai, B. Jiang, A. Rusydi, J. N. Eckstein, S. D. Bader, and J. M. Zuo, Phys. Rev. Lett. 99, 196404 (2007).

${ }^{6}$ S. J. May, A. B. Shah, S. G. E. te Valthuis, M. R. Fitzsimmons, J. M. Zuo, X. Zhai, J. N. Eckstein, S. D. Bader, and A. Bhattacharya, Phys. Rev. B 77, 174409 (2008).

${ }^{7}$ T. Satoh, K. Miyano, Y. Ogimoto, H. Tamaru, and S. Ishihara, Phys. Rev. B 72, 224403 (2005).

${ }^{8}$ J. J. U. Buch, G. Lalitha, T. K. Pathak, N. H. Vasoya, V. K.
Lakhani, P. V. Reddy, R. Kumar, and K. B. Modi, J. Phys. D 41, 025406 (2008).

${ }^{9}$ O. K. Andersen and O. Jepsen, Phys. Rev. Lett. 53, 2571 (1984).

${ }^{10}$ A. J. Millis, Phys. Rev. B 55, 6405 (1997).

${ }^{11}$ J.-S. Zhou and J. B. Goodenough, Phys. Rev. B 68, 054403 (2003).

${ }^{12}$ S. Satpathy, Z. S. Popovic, and F. R. Vukajlovic, Phys. Rev. Lett. 76, 960 (1996).

${ }^{13}$ D. Feinberg, P. Germain, M. Grilli, and G. Seibold, Phys. Rev. B 57, R5583 (1998).

${ }^{14}$ P. W. Anderson and H. Hasegawa, Phys. Rev. 100, 675 (1955).

${ }^{15}$ C. Zener, Phys. Rev. 82, 403 (1951).

${ }^{16}$ P.-G. De Gennes, Phys. Rev. 118, 141 (1960).

${ }^{17}$ B. R. K. Nanda, S. Satpathy, and M. S. Springborg, Phys. Rev. Lett. 98, 216804 (2007).

${ }^{18}$ H. Meskine, H. Konig, and S. Satpathy, Phys. Rev. B 64, 094433 (2001). 